\title{
Behavior of passive admixture in a vortical hydrodynamic field
}

\author{
R.O.Bobrov ${ }^{1}$, A.V.Kyrylyuk ${ }^{2}$, A.V.Zatovsky ${ }^{1}$ \\ 1 Department of Theoretical Physics, \\ Odessa National Mechnikov University, \\ 2, Dvoryanskaya Str., 65026 Odessa, Ukraine \\ 2 Eindhoven University of Technology, \\ P.O.Box 513, 5600 MB Eindhoven, The Netherlands
}

Received July 18, 2005

\begin{abstract}
The motion of passive admixture of spherical particles in the stationary hydrodynamic field of a swirling flow is studied. A spherical particle of a given mass in the hydrodynamic field of a swirling flow is located on a certain circular orbit, where the centrifugal force is compensated by the radial drag force due to the sink. This leads to the separation of the host fluid and admixture. A theory of Brownian motion of admixture in dilute solutions with a non-uniform flow is constructed.
\end{abstract}

Key words: vortical hydrodynamic field, Brownian motion, passive admixture

PACS: $47.32 .-y, 82.40 . C k, 02.60 . C b$

\section{Introduction}

At present, there are many studies that try to explain the behavior of admixture or microorganisms in various hydrodynamic fields (see for example [1-4]). Much attention has been devoted to stochastic fields [1,2] and external noise [6,7]. It is known that if passive admixture is subjected to a stochastic hydrodynamic field, then the foreign particles form clusters. In 1970-1971 in Argentina, 500 balloons of the same density were released [5]. They spread over the entire southern hemisphere on the height about $12 \mathrm{~km}$. This modeling showed that the balloons were concentrated in groups corresponding to a clasterization process. The mathematical model demonstrated that the balloons moved in regular and stochastic hydrodynamic fields. Interaction between particles typically leads to a bound state in a system of particles. Apart from this, a spatially non-uniform distribution of both interacting and non-interacting particles in the velocity field of a hydrodynamic flow is possible. The problems of this kind attract much attention (see for example [6] and references therein). In the present study, we consider the motion of non-interacting foreign particles in the hydrodynamic field of a swirling flow. A separate particle in the field of a swirling flow moves in a planar circular orbit [8]. In this orbit, the angular and radial velocities of the stream are inversely proportional to the distance from the axis of the drain, so that the centrifugal force is compensated by the radial drag force due to the sink. Such an orbit appears to be steady only for the field of a swirling flow, for which the angular and radial velocities of the stream are inversely proportional to the distance from the particle to the center of the drain. However, this cannot be the case for other hydrodynamic fields. In the presence of random sources passive admixture can be considered as a set of Brownian particles. In this case, the most probable position of the particles is an equilibrium orbit. 


\section{The motion of particles in a hydrodynamic field}

We consider the motion of non-interacting particles of identical mass in the vortical hydrodynamic field of a rotating cylindrical chamber of radius $R$ :

$$
U_{r}=-\frac{V}{r} R, \quad U_{\varphi}=\frac{\omega R^{2}}{r}, \quad r \geqslant R .
$$

This field is the solution of the hydrodynamical problem of steady rotation of the infinite porous cylinder in a motionless fluid with the angular velocity $\omega$ and the speed of the suction $V$ on its border. We suppose that each particle in the stream experiences only Stokes' force and that the particles do not affect the stream. The planar motion of any particle in the stream is described by the equation

$$
\dot{\vec{V}}=-\lambda(\vec{V}-\vec{U})
$$

Here, $\vec{V}$ is the velocity of the particle, $\lambda=6 \pi \eta d / M, \eta$ is the kinematic viscosity, $d$ is the radius of the particle, and $M$ is the mass of the particle. In the polar reference frame the equations of motion have the following form:

$$
\ddot{r}-r \dot{\varphi}^{2}=-\lambda\left(\dot{r}+\frac{a}{r}\right), \quad r \ddot{\varphi}+2 \dot{r} \dot{\varphi}=-\lambda\left(r \dot{\varphi}-\frac{b}{r}\right)
$$

where $a=V R$ and $b=\omega R^{2}$. After multiplying the second equation in equations (3) by $r$, introducing a new variable $\psi=r^{2} \dot{\varphi}$, and making some mathematical transformations we obtain

$$
\ddot{r}-\frac{\psi^{2}}{r^{3}}=-\lambda\left(\dot{r}+\frac{a}{r}\right), \quad \dot{\psi}=-\lambda(\psi-b),
$$

where

$$
\psi=b+C \exp (-\lambda t)
$$

Here, $C$ is the constant of integration, which is determined by the initial conditions. Using the solution of equations (4) one can reduce equations (3) to a single equation

$$
\ddot{r}+\lambda \dot{r}-f(r)=F(t) / r^{3}
$$

with

$$
f(r)=-\frac{\lambda a}{r}+\frac{b^{2}}{r^{3}}, \quad F(t)=2 b C \exp (-\lambda t)+C^{2} \exp (-2 \lambda t) .
$$

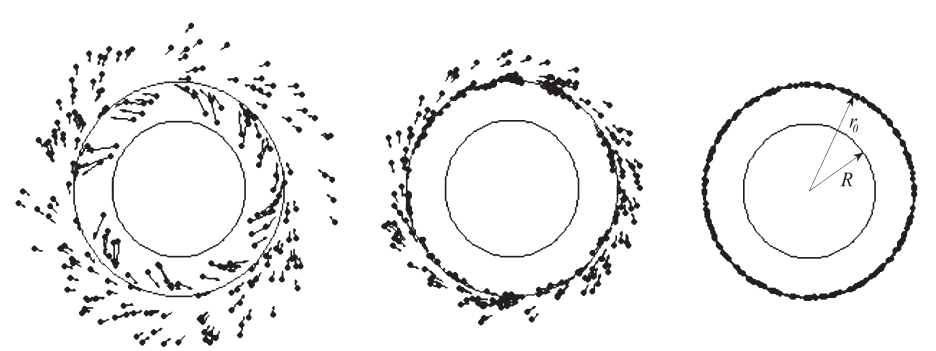

Figure 1. The distribution of non-interacting spherical particles $(\mathrm{N}=200)$ in the hydrodynamic field of a swirling flow at different times. The circle of radius $r_{0}$ represents the equilibrium orbit.

In the following we suppose that the friction in the system is large, $\lambda t \gg 1$. In other words, we consider the motion of the particles at times which are bigger than characteristic hydrodynamic times, $t \gg 1 / \lambda$. The term in equations (4) which contains $\ddot{r}$ has the magnitude of the order of $U_{r} / t$ and $\lambda \dot{r} \approx \lambda U_{r}$. The ratio of the first term to the second one in equations (5) is $\lambda t$ and therefore the first term, which is an inertial term, can be omitted. Since the function $F(t) \sim \exp (-\lambda t)$, the 
term in the right part of equations (5) decreases more rapidly than the inertial term. As a result, in this approximation the equation of motion turns into

$$
\lambda \dot{r}-f(r)=0
$$

Let us consider the stationary motion, when the radial velocity of a particle stays constant in time and is equal to zero. Then equations (6) read as follows $f(r)=0$ or $-\lambda a / r+b^{2} / r^{3}=0$. From this equation it follows that a particle moves along an equilibrium orbit of radius

$$
r_{0}=\frac{b}{\sqrt{\lambda a}}
$$

which is entirely determined by the vortical field and Stokes' friction. The obtained numerical solution of equations (3) evidently shows a trend for the motion of the particles toward the equilibrium orbit, figure 1.

For simplicity, all the particles are uniformly distributed in the field equations (1) with the initial coordinates and velocities equal in module to those of the stream at these positions:

$$
\left.r(t)\right|_{t=0}=r(0),\left.\quad \varphi(t)\right|_{t=0}=\varphi(0),\left.\quad \dot{r}(t)\right|_{t=0}=-\frac{V R}{r(0)},\left.\quad \dot{\varphi}(t)\right|_{t=0}=\frac{\omega R^{2}}{r^{2}(0)} .
$$

The time needed to reach an equilibrium orbit is entirely determined by the initial positions of the particles. The dependence of the radial coordinate on the number of time steps is represented in figure 2. This dependence is shown for five particles with different initial coordinates. Since the angular component of the particle velocities is determined purely by the angular velocity of the stream and the radial coordinate, the centripetal acceleration and the field of the swirling flow make a bigger effect on the motion of a particle at smaller distances to the drain. With the increase in the distance this effect decreases inversely proportionally to the coordinate. Consequently, the time needed to reach an equilibrium orbit is determined purely by the initial position. If a particle is closer to the drain, then it comes to an equilibrium orbit faster, which is demonstrated in figure 2.

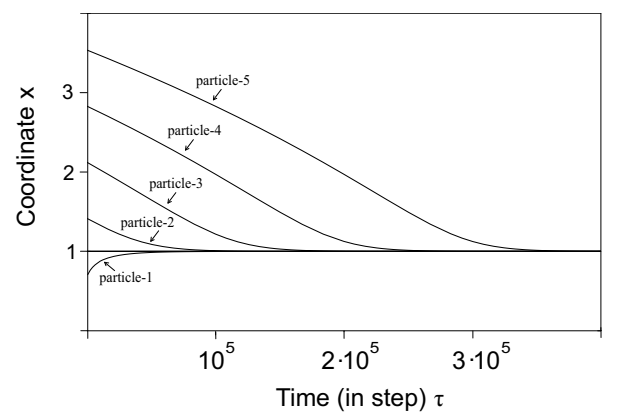

Figure 2. The dependence of the dimensionless radial coordinate $x=r / r_{0}$ on the number of time steps $\tau=t \lambda(\mathrm{N}=5), \beta=\omega / \lambda=10^{-2}, \gamma=V /(\lambda R)=5 \cdot 10^{-5}$.

The hydrodynamic field studied can model a hurricane in the air. Based on the results of the present study one can conclude that all the objects sucked by the hurricane will not leave the funnel of a hurricane under fixed conditions. From equations (7) it follows that the objects of different mass will rotate in different orbits. The radius of an equilibrium orbit is proportional to the square root of the mass of the object. This means that heavy particles rotate in equilibrium orbits of a bigger radius. Similarly, our results can be applied to the analysis of the fish schooling in such a hydrodynamic field. The motion of large predator fish can cause the appearance of a swirling flow similar to the one studied in the present work.

The condition for a particle not to be sucked within the rotating cylinder (see figure 2) is given by the inequality 


$$
r_{0}>R, \quad \omega>\sqrt{\frac{6 \pi \eta a V}{M R}} .
$$

After introducing the dimensionless constants

$$
\beta=\frac{\omega}{\lambda}, \quad \gamma=\frac{V}{\lambda R},
$$

equations (8) can be rewritten in a dimensionless form with the following constraint on these constants:

$$
\frac{\beta}{\sqrt{\gamma}}>1
$$

From the physical point of view the function $f(r)$ in equations (5) and equations (6) can be interpreted as an additional force which acts on the particle. This function contains information about the centripetal acceleration and the field of the swirling flow equations (1). Since in case of $\lambda t \gg 1$ the decay of the function $F(t)$ is much faster than the decay of the inertial terms, equations (5) can be rewritten as

$$
\ddot{r}+\lambda \dot{r}-f(r)=0 .
$$

This equation can be interpreted as the equation of motion of the particle in the effective potential field $\Phi(r)$ :

$$
\begin{aligned}
f(r) & =-\frac{\partial}{\partial r} \Phi(r) \\
\Phi(r) & =\lambda a \ln (r)+\frac{b^{2}}{r^{2}} \frac{1}{2}+\text { const. }
\end{aligned}
$$

Since the constant in the expression for the effective potential energy is arbitrary, we have chosen

$$
\Phi(r)=\lambda a \ln \left(\frac{r}{r_{0}}\right)+\frac{b^{2}}{r^{2}} \frac{1}{2} .
$$

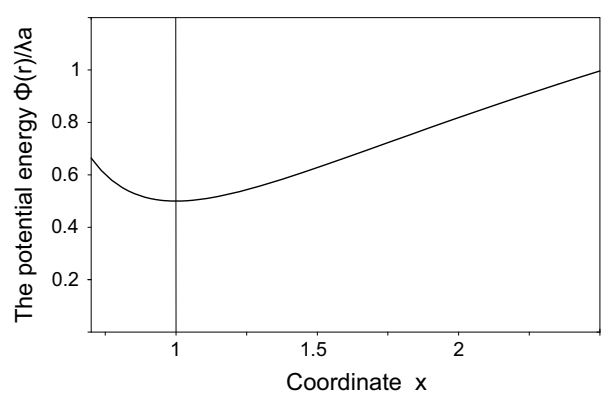

Figure 3. The dependence of the potential energy on the dimensionless coordinate $x=r / r_{0}$. Here $\beta=10^{-2}$ and $\gamma=5 \cdot 10^{-5}$.

In figure 3 the potential energy as a function of the dimensionless coordinate $x=r / r_{0}$ is drawn. The minimum of the energy is at $x=1$, which corresponds to the fact that the most probable location of the particle is in the equilibrium orbit. The detailed analysis shows that in the vicinity of the minimum, the equilibrium orbit represents a steady state. The potential energy has an asymptote $\left.\Phi(x)\right|_{x \rightarrow \infty} \rightarrow \infty$. This suggests that independently of the energy each particle eventually reaches the equilibrium orbit. 


\section{Brownian motion of particles}

The particles in the hydrodynamic field of the swirling flow can be considered as Brownian particles [9]. In this case the stochastic force should be added to the equation of motion equations (5), so that the corresponding Langevin equation reads

$$
\ddot{r}+\lambda \dot{r}-f(r)=n(t), \quad \dot{r}=V_{r} .
$$

Let us suppose that the medium is in equilibrium and that no external force is present. Then, all directions of the stochastic force are equivalent and therefore its mean value is equal to zero:

$$
\langle n(t)\rangle=0
$$

We also suppose that the characteristic time of the correlations of the Langevin force, $\tau_{\text {cor }}$, is much smaller than the hydrodynamic relaxation time, $\tau_{\text {rel }}=1 / \lambda$. In the first-order approximation this characteristic time is set to zero. Since no direction is more preferable than others, different components of the Langevin force do not correlate with one another (collisions far in time are statistically independent):

$$
\left\langle n(t) n\left(t^{\prime}\right)\right\rangle=2 D \delta\left(t-t^{\prime}\right),
$$

where $2 D$ is the intensity of the Langevin source, which has the meaning of the average intensity of the random collisions with atoms of the medium. As $\tau_{\text {cor }} \ll \tau_{\text {rel }}$, the stochastic process can be considered as a Gaussian one. Due to the large friction coefficient, the change in coordinates takes place in two stages. First, the Maxwell distribution of velocities $V_{r}$ is rapidly established and then the considerably slower process of establishing the Boltzmann distribution over the coordinates $r$ occurs. We consider only the second stage of slow development of the equilibrium. Such a process is described by the reduced Langevin equation

$$
\lambda \dot{r}-f(r)=n(t) .
$$

The corresponding Einstein-Fokker-Planck equation for the probability of the particle to leap from one state to another can be written as follows:

$$
\frac{\partial W}{\partial t}=-\frac{1}{\lambda} \frac{\partial}{\partial r} f(r) W+D \frac{\partial^{2} W}{\partial r^{2}} .
$$

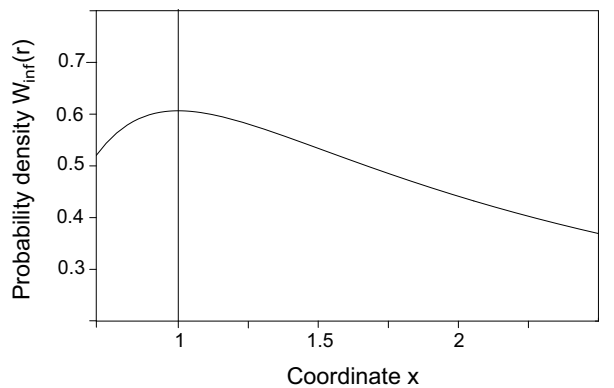

Figure 4. The dependence of the probability density on the dimensionless coordinate $x=r / r_{0}$. Here $\beta=10^{-2}$ and $\gamma=5 \cdot 10^{-5}$.

Within the framework of the assumptions made, it is possible for the system of Brownian particles in liquid medium to relax to an equilibrium state. In particular, the probability distribution function relaxes to the Boltzmann distribution which is given by the stationary solution of equations (13):

$$
W_{\text {inf }}(r)=C \exp \left[\frac{1}{\lambda D} \int f(r) \mathrm{d} r\right] \sim \exp \left[-\frac{1}{\lambda D} \Phi(r)\right]
$$


or

$$
W_{\mathrm{inf}}(r) \sim \exp \left[-\frac{a}{D}\left(\ln \left(\frac{r}{r_{0}}\right)+\frac{1}{2}\left(\frac{r_{0}}{r}\right)^{2}\right)\right] .
$$

In figure 4 the dependence of the probability density on the dimensionless coordinate $x=r / r_{0}$ is depicted. From figure 4 one can see that the maximum of this function corresponds to the equilibrium orbit of radius $r_{0}$. Therefore, the numerical modeling shows that both representations of the particles, either Brownian particles or passive admixture, lead to the maximum probability of finding the particles in the equilibrium orbit.

\title{
4. Conclusion
}

We have investigated the motion of passive admixture of spherical particles in a stationary hydrodynamic field of a swirling flow. The analysis shows that the most probable position of a foreign particle is along an equilibrium orbit which is determined by the characteristics of the vortical field. Considering the particles as Brownian ones, the distribution of the particles in the swirling flow in a stationary regime is found.

\section{References}

1. Klyatskin V.I., Physics-Uspekhi, 2003, No. 7, 173, 689.

2. Klyatskin V.I., Koshel' K.V., Physics-Uspekhi, 2000, No. 7, 170, 771.

3. Ivanitsky G.R., Medvinsky A.B., Tsyganov M.A., Physics-Uspekhi, 1994, No. 10, 164, 1041.

4. Ivanitsky G.R., Medvinsky A.B., Tsyganov M.A., Physics-Uspekhi, 1991, No. 4, 161, 13.

5. Mesinger F., Mintz Y., Physics-Uspekhi, Los Angeles: Dep. Meteorology, Univ. of California, Tech. Rep., 1970, 4, 5 .

6. Czirok A., Vicsek T., Physica A, 2000, 281, 17.

7. Horsthemke W., Lefever R. Noise-induced Transitions. Springer-Verlag, Berlin, 1984.

8. Goldshtik M.A. Vortex Flows. Nauka, Novosibirsk, 1981 (in Russian)

9. Reimann P., Phys. Reports, 2002, 361, 57.

\section{Поведінка пасивної домішки у вихровому гідродинамічному полі}

\author{
Р.О.Бобров ${ }^{1}$, А.В.Кирилюк ${ }^{2}$, А.В.Затовський \\ 1 Одеський національний університет ім.І.І.Мечникова, \\ факультет теоретичної фізики \\ 65026 Одеса, вул. Дворянська, 2, Україна \\ 2 Технологічний університет Айндховену, \\ 5600 МВ Айндховен, Нідерланди
}

Отримано 18 липня 2005 р.

Досліджується рух пасивної домішки сферичних частинок у стаціонарному гідродинамічному полі потоку, що крутиться. СФерична частинка заданої маси у гідродинамічному полі потоку, що крутиться, $є$ розташована на певній коловій орбіті, де відцентрова сила компенсується радіальною силою, що викликана стоком. Це приводить до розділення основної компоненти рідини та домішки. Розвивається теорія броунівського руху домішки у слабких розчинах з неоднорідним потоком.

Ключові слова: вихрове гідродинамічне поле, броунівський рух, пасивна домішка

PACS: 47.32.-y, 82.40.Ck, 02.60.Cb 